\title{
HMPAO SPECT in Parkinson's disease before and after levodopa: correlation with dopaminergic responsiveness
}

\author{
H S Markus, D C Costa, A J Lees
}

\begin{abstract}
Regional cerebral perfusion was evaluated by SPECT with technetium $99 \mathrm{~m}$ hexamethylpropyleneamine oxime (99mTc HMPAO) as a tracer in 21 patients presenting with Parkinson's disease and in 11 normal controls. In the parkinsonian patients, scans were performed both off treatment, and after levodopa, and clinical dopaminergic responsiveness was evaluated. Uptake of HMPAO by the basal ganglia was significantly decreased in the parkinsonian subjects, compared with normal controls. This reduction was seen in both responders $(n=14)$ and non-responders $(n=7)$ to dopaminergic treatment. Uptake of HMPAO by the basal ganglia rose after treatment with levodopa, but the change was similar in both responders and non-responders. $B y$ contrast a striking difference in cortical HMPAO uptake was found between responders and non-responders, with significantly lower uptake in the medial temporal and posterior parietal cortex in the non-responders. This reduction was symmetrical. Basal ganglia perfusion assessed by this technique is unlikely to be of use in the diagnosis of Parkinson's disease that is responsive to dopaminergic treatment. The presence of extensive cortical involvement on a baseline scan correlates with a lack of dopaminergic responsiveness, however, and this may be useful diagnostically.
\end{abstract}

$(\Im$ Neurol Neurosurg Psychiatry 1994;57:180-185)

The discovery that neuroprotective therapy may be effective in delaying the progression of early idiopathic Parkinson's disease ${ }^{1}$ has reinforced the need for tests aiding early diagnosis. Whereas conventional structural imaging is usually normal, functional imaging shows abnormalities early in the disease. In the striatum reduced glucose metabolism and impaired uptake of $\left({ }^{18} \mathrm{~F}\right)$ fluorodopa has been shown by positron emission tomography (PET). ${ }^{23}$ With PET a decrease in basal ganglia blood flow has also been shown ${ }^{4}$; this is believed to result from the coupling of cerebral metabolism and blood flow, and to reflect lower metabolism in the affected basal ganglia. Although ${ }^{18} \mathrm{~F}$-fluorodopa PET offers the most sensitive technique for identifying early Parkinson's disease and differentiating it from other extrapyramidal syndromes, ${ }^{3}$ it is not available on a large enough scale to provide a routine method of diagnosis. SPECT is more widely available; use of the tracer technetium-99m hexamethylpropyleneamine oxime ( ${ }^{99 m}$ Tc HMPAO) allows semiquantitative imaging of regional cerebral blood flow. ${ }^{5}$ ${ }^{99} \mathrm{~m}$ Tc HMPAO is a lipophilic compound that easily crosses the blood brain barrier and is therefore taken up on first pass through the brain where it is retained for several hours. ${ }^{5}$ It is distributed in proportion to regional cerebral blood flow. ${ }^{5}$ The technique requires only an intravenous injection, and with modern imaging equipment scanning times are as short as 15 minutes. ${ }^{6}$ With this technique reduced regional blood flow has been found in the basal ganglia in parkinsonian patients, and it has been reported that this returns towards normal after treatment with dopaminergic drugs. ${ }^{7}$ It has been suggested that these findings might represent a useful diagnostic test for Parkinson's disease. Studies have also shown that blood flow increases in the basal ganglia of normal subjects after dopaminergic stimulation, ${ }^{8}$ although the increase seen may be less than that occurring in Parkinson's disease.

Even among subjects with a clinical syndrome resembling Parkinson's disease as many as a quarter of patients do not have the disease on pathological examination ${ }^{90}$; misdiagnoses include multiple system atrophies, Alzheimer's type pathology, progressive supranuclear palsy, corticobasal degeneration, and vascular disease. ${ }^{10}$ These alternative diagnoses tend to have cortical involvement to a greater degree than that seen in Parkinson's disease. Regional cerebral blood flow imaging might differentiate these cases from Parkinson's disease.

For these reasons we have carried out a study of ${ }^{99 m} \mathrm{Tc}$ HMPAO SPECT in Parkinson's disease. We have compared scans from patients with Parkinson's disease on no treatment with scans from normal controls; in patients with Parkinson's disease we have compared baseline scans on no treatment with SPECT studies after a single oral dose of levodopa. In particular we have examined whether basal ganglia abnormalities are useful in detecting patients responsive to levodopa, and distinguishing them from non-parkinsonian subjects and from non-responders. We have also investigated whether cortical abnormalities are more frequent in patients unresponsive to levodopa than in patients with classical levodopa responsive disease. 
Methods

SUBJECTS

Twenty-one patients presenting to a neurology department with a parkinsonian syndrome clinically thought to be Parkinson's disease were studied. All subjects fulfilled the United Kingdom Parkinson's Disease Society brain bank clinical criteria. ${ }^{10}$ No subjects had clinical evidence of dementia and all were able to answer all questions on the mini mental test questionnaire correctly. In all subjects dopaminergic responsiveness was assessed by studying the effect of an oral dose of $250 \mathrm{mg} / 25 \mathrm{mg}$ carbidopa levodopa (Sinemet 275). All dopaminergic drugs were withheld for 48 hours before testing and before baseline SPECT scanning. Before and 90 minutes after the dose, patients were assessed in three ways: by scoring on a modified Webster's score, ${ }^{11}$ by the time taken to walk a $12 \mathrm{~m}$ distance (mean of two attempts), and by an alternate unilateral hand tapping for $30 \mathrm{sec}-$ onds on digital counters mounted $20 \mathrm{~cm}$ apart (mean of two trials for each hand). A test was judged positive if there was a $>15 \%$ increase in tapping time for either hand, a $>25 \%$ improvement in walking time, or an improvement in Webster's score of 3 or more, according to previously published guidelines. ${ }^{12}$ In patients who failed to respond to an acute dose of levodopa a one month trial of levodopa (100 mg three times a day) was instituted, and patients reassessed after this.

Eleven normal controls (patients' relatives and hospital staff) with no neurological, vascular, or other relevant medical history, and no evidence of cognitive impairment, were studied.

\section{SPECT SCANNING}

Subjects were injected with approximately $500 \mathrm{MBq}$ of ${ }^{99 \mathrm{~m}}$ Tc HMPAO into the antecubital vein of one arm while sitting with their eyes open in a quiet room. Scans were then performed after 7-10 minutes. Subjects lay supine in the GE/CGR Neurocam, a braindedicated three-headed camera SPECT system. ${ }^{7}$ The data acquisition indices were: high resolution collimators, 128 views, $64 \times 64$ matrix ( $4 \mathrm{~mm}$ pixel size), 20 seconds per view (14 minute total acquisition time). Using this system with similar acquisition indices, the reconstructed spatial resolution in water at the centre of the field of view is $9 \cdot 7$ mm. ${ }^{7}$ Transaxial tomographic slices, $4 \mathrm{~mm}$ thick, were reconstructed by means of a Hanning prefilter with 1.0 cycles/cm cut off frequency, a ramp backprojection filter, and attenuation correction, assuming a uniform linear attenuation coefficient in the brain equal to $0.12 \mathrm{~cm}^{-1}$. Horizontal slices (parallel to the anterior commissure-posterior commissure line) as well as sagittal and coronal slices, $8 \mathrm{~mm}$ thick, were obtained by interpolation. Analysis was performed both visually and quantitatively.

Visual analysis

SPECT scans of controls and of patients with
Parkinson's disease on no treatment, were analysed qualitatively. Blinded to the diagnosis the cortical uptake was graded as normal (score 0 ), or showing mild (score 1 ), or severe (score 2) deficits.

\section{Semiquantitative analysis}

Both pre and posttreatment scans were analysed. ${ }^{99 \mathrm{~m}}$ Tc HMPAO uptake in individual brain areas was quantified by placing square $4 \times 4$ pixel regions of interest, corresponding to $16 \times 16 \times 8 \mathrm{~mm}^{3}$ brain volumes, on positions standardised with reference to a stereotactic brain atlas. ${ }^{13}$ These consisted of the following numbers of regions of interest on each brain region: cerebellum nine, basal ganglia three, caudate one, putamen two, thalamus two, prefrontal region three, anterior frontal eight, posterior frontal three, anterior parietal three, posterior parietal three, superior parietal six, inferior parietal six, lateral temporal nine, medial temporal nine, occipital nine, all bilaterally, and brainstem one. All quantitative analysis was performed by one observer blinded to treatment state. Intraobserver reproducibility of this method of measuring regional uptake was assessed by repeating the counting procedure on two separate days for five scans (10 cerebral hemispheres). The mean (SD) absolute percentage difference in counts for each region was: cerebellum $0.35(0.38)$, mean basal ganglia $0.85(0.95)$, thalamus 0.14 $(0.40)$, occipital cortex $0.93(1.37)$, medial temporal cortex $1.39(0.85)$; lateral temporal cortex $0.69(0.65)$, anterior frontal cortex $1.01(0.84)$, posterior frontal cortex 1.12 (0.75), superior parietal cortex $0.98(0.61)$, inferior parietal cortex $0.99(0.90)$, anterior parietal cortex $1.23(1.40)$, posterior parietal cortex $1.00(0.94)$, and brainstem 0.02 $(0.04)$.

The mean count per pixel per brain region was calculated for each brain region on both left and right sides. Static HMPAO SPECT does not allow absolute quantification of regional cerebral blood flows. Semi quantitative analysis can be performed by comparing uptake in the area under investigation with uptake in an area unaffected by the disease process. The cerebellum is pathologically relatively spared in Parkinson's disease and therefore it is commonly usually used as the reference region in SPECT studies in Parkinson's disease. ${ }^{71415}$ We expressed HMPAO uptake for each region as the ratio of uptake in that region to that in the cerebellum. As there were no significiant differences between uptake in the left and right hemispheres, both sides were included together in comparisons. To determine irregularities in uptake quantitatively, percent interhemispheric asymmetry indices were calculated, for each brain area, as the absolute value of 200 (right - left)/(right + left).

Comparisons between the two groups, controls, and all subjects with Parkinson's disease, were made with Student's $t$ test. Comparisons between the three groups, controls, responders with Parkinson's disease and 
non-responders with Parkinson's disease were made by analysis of variance followed by Scheffe's test for multiple comparisons to identify differences between individual groups. Changes after levodopa in the two Parkinson's disease groups were made with a paired $t$ test. The Wilcoxon signed rank test was used to compare the severity of cortical involvement in the different groups. Significance was taken as the $\mathrm{p}<0.05$ level.

\section{Results}

CLINICAL DETAILS

Twenty-one patients with Parkinson's disease were studied. Mean (SD) age was $64 \cdot 0(10 \cdot 6)$ years for patients with Parkinson's disease and $70.7(6.3)$ years for normal controls (NS). The male to female ratio was $13: 8$ for patients with Parkinson's disease and 6:5 for normal controls. Twelve of the 21 parkinsonian patients had never been on medication for their Parkinson's disease; the other nine had been on treatment for two or less years. The Hoehn and Yahr scale ${ }^{16}$ was I in eight and II in 13 subjects.

Fourteen of the 21 patients responded to levodopa according to the criteria described in the methods section. In this group mean (SD) Webster's score before treatment was $7 \cdot 8(2.3)$ and this decreased to $2.4(1.5)$ after levodopa. In the responder group mean (SD) walking time before treatment was $15.5(6 \cdot 3)$ seconds and after levodopa treatment there was a mean (SD) percentage decrease in walking time of $18.4(8.6) \%$. Mean (SD) tapping score was $71.4(26.3)$ taps, and after levodopa there was a mean (SD) percentage increase in tapping score of $24 \cdot 2(12 \cdot 1) \%$ The seven non-responders showed positive responses on none of the three criteria; of these seven, five showed little response on any of the three tests, and in two walking time and tapping time definitely improved but not enough to meet the criteria defined in the protocol. In the non-responders mean (SD) Webster's score was 7.0 (5.0) before and $6.7(4.9)$ after treatment with levodopa. In this group mean (SD) walking time was
$18 \cdot 1(27 \cdot 3)$ seconds and after there was a mean (SD) percentage decrease in walking time of $4.0(8.0) \%$. Tapping score was 60.3 (23.6) before treatment, and after levodopa there was a mean (SD) percentage decrease in tapping time of $5.5(7 \cdot 7) \%$. All seven nonresponders received a one month trial of levodopa at a dose of $100 \mathrm{mg}$ three times a day; none showed an improvement in Webster's score after this treatment.

Of the 14 responders, five had presented with a tremor, eight with symptoms attributable to bradykinesia/rigidity, and one presented with both types of symptoms. Of the seven non-responders two presented with tremor, three with symptoms attributable to bradykinesia/rigidity, and one with both types of symptoms. There was no obvious clinical difference between the two groups except that more of the non-responders had bilateral symptoms (6/7 compared with $7 / 14 ; \chi^{2} \mathrm{NS}$ ). The non-responders were older than the responders (mean (SD) 69.1 (11.5) $v$ 61.4 (9.4) years) but this difference was not significant. Duration of symptoms was not different between the two groups (responders mean (SD) 2.06 (1.72); non-responders 1.29 (0.70 years). All non-responders had CT head scans performed; these were normal in three, and showed mild atrophy in three cases and mild periventricular hypodensity in one case. Five of the responders had computed tomography scans; these were normal in four cases and showed mild atrophy in one case.

\section{VISUAL ANALYSIS}

The mean grade of cortical deficits was greater in subjects with Parkinson's disease than in normal controls $(2.14 \quad v \quad 1.61$; Wilcoxon; $\mathrm{p}<0.004)$. Within the Parkinson's disease group severe cortical perfusion deficits were more common in nonresponders (7/7) than in responders (3/14) $\left(\chi^{2}, \mathrm{p}<0.05\right)$. The mean grade of cortical deficit was higher in non-responders (3.0), than in responders $(1 \cdot 71$; Wilcoxon $\mathrm{p}<0.05)$, or than in normal subjects $(1.61$; Wilcoxon $\mathrm{p}<0.03$ )

Table Mean (SD) regional cerebral blood flow measured by HMPAO SPECT in different cerebral regions

\begin{tabular}{|c|c|c|c|c|c|c|c|c|c|c|}
\hline & $\begin{array}{l}\text { Basal } \\
\text { ganglia }\end{array}$ & Putamen & Caudate & Thalamus & $\begin{array}{l}\text { Brain } \\
\text { stem }\end{array}$ & $\begin{array}{l}\text { Pre } \\
\text { frontal }\end{array}$ & $\begin{array}{l}\text { Anterior } \\
\text { frontal }\end{array}$ & $\begin{array}{l}\text { Posterior } \\
\text { frontal }\end{array}$ & $\begin{array}{l}\text { Superior } \\
\text { parietal }\end{array}$ & $\begin{array}{l}\text { Inferior } \\
\text { parietal }\end{array}$ \\
\hline $\begin{array}{l}\text { Controls } \\
(\mathbf{n}=11)\end{array}$ & $\begin{array}{l}0.956 \\
(0.047)\end{array}$ & $\begin{array}{l}0.968 \\
(0.078)\end{array}$ & $\begin{array}{l}0.905 \\
(0.078)\end{array}$ & $\begin{array}{l}0.925 \\
(0.069)\end{array}$ & $\begin{array}{l}0.813 \\
(0 \cdot 046)\end{array}$ & $\begin{array}{l}0.755 \\
(0.039)\end{array}$ & $\begin{array}{l}0.774 \\
(0.049)\end{array}$ & $\begin{array}{l}0.789 \\
(0.045)\end{array}$ & $\begin{array}{l}0.823 \\
(0.057)\end{array}$ & $\begin{array}{l}0.776 \\
(0.052)\end{array}$ \\
\hline $\begin{array}{l}\text { PD all } \\
(\mathrm{n}=21)\end{array}$ & $\begin{array}{l}0.906 \\
(0.060)\end{array}$ & $\begin{array}{l}0.914 \\
(0.078)\end{array}$ & $\begin{array}{l}0.859 \\
(0.053)\end{array}$ & $\begin{array}{l}0.926 \\
(0.056)\end{array}$ & $\begin{array}{l}0.810 \\
(0.050)\end{array}$ & $\begin{array}{l}0.726 \\
(0.049)\end{array}$ & $\begin{array}{l}0.737 \\
(0.040)\end{array}$ & $\begin{array}{l}0.748 \\
(0.038)\end{array}$ & $\begin{array}{l}0.765 \\
(0.051)\end{array}$ & $\begin{array}{l}0.745 \\
(0.048)\end{array}$ \\
\hline $\begin{array}{l}\text { PD-RES } \\
(\mathrm{n}=14)\end{array}$ & $\begin{array}{l}0.913 \\
(0.047)\end{array}$ & $\begin{array}{l}0.923 \\
(0.048)\end{array}$ & $\begin{array}{l}0.858 \\
(0.037)\end{array}$ & $\begin{array}{l}0.926 \\
(0.041)\end{array}$ & $\begin{array}{l}0 \cdot 820 \\
(0 \cdot 043)\end{array}$ & $\begin{array}{l}0.739 \\
(0.046)\end{array}$ & $\begin{array}{l}0.746 \\
(0.039)\end{array}$ & $\begin{array}{l}0.749 \\
(0.040)\end{array}$ & $\begin{array}{l}0.771 \\
(0.049)\end{array}$ & $\begin{array}{l}0.745 \\
(0.043)\end{array}$ \\
\hline \multirow[t]{2}{*}{$\begin{array}{l}\text { PD-NR } \\
(\mathrm{n}=7)\end{array}$} & $\begin{array}{l}0.892 \\
(0.080)\end{array}$ & $\begin{array}{l}0.895 \\
(0.081)\end{array}$ & $\begin{array}{l}0.861 \\
(0.079)\end{array}$ & $\begin{array}{l}0.926 \\
(0.079)\end{array}$ & $\begin{array}{l}0.790 \\
(0.061)\end{array}$ & $\begin{array}{l}0.702 \\
(0.048)\end{array}$ & $\begin{array}{l}0.719 \\
(0.035)\end{array}$ & $\begin{array}{l}0.736 \\
(0.041)\end{array}$ & $\begin{array}{l}0.754 \\
(0.053)\end{array}$ & $\begin{array}{l}0.745 \\
(0.057)\end{array}$ \\
\hline & & & & & p Values & & & & & \\
\hline $\begin{array}{l}v \text { C } \\
\text { PD-RES }\end{array}$ & 0.004 & 0.004 & 0.009 & NS & NS & 0.020 & 0.002 & 0.0001 & 0.0001 & 0.017 \\
\hline $\begin{array}{c}v \mathrm{C} \\
\text { PD-NR }\end{array}$ & 0.05 & 0.017 & 0.05 & NS & NS & NS & NS & 0.02 & 0.005 & NS \\
\hline $\begin{array}{c}v \mathrm{C} \\
\text { PD-RES }\end{array}$ & 0.02 & 0.02 & NS & NS & NS & 0.004 & 0.002 & 0.004 & 0.002 & NS \\
\hline$v$ PD-NR & NS & NS & NS & NS & NS & 0.05 & NS & NS & NS & NS \\
\hline
\end{tabular}

$\mathrm{C}=$ Controls; $\mathrm{PD}=$ subjects with Parkinson's disease; $\mathrm{PD}-\mathrm{RES}=$ responders to dopamine; PD-NR = non-responders; $\mathrm{HMPAO}$ uptake is expressed as the ratio to cerebellar uptake; $\mathrm{p}$ values for $\mathrm{PD}$ all $v \mathrm{C}$ from Students' $t$ test; other comparisons by Scheffe's test. 


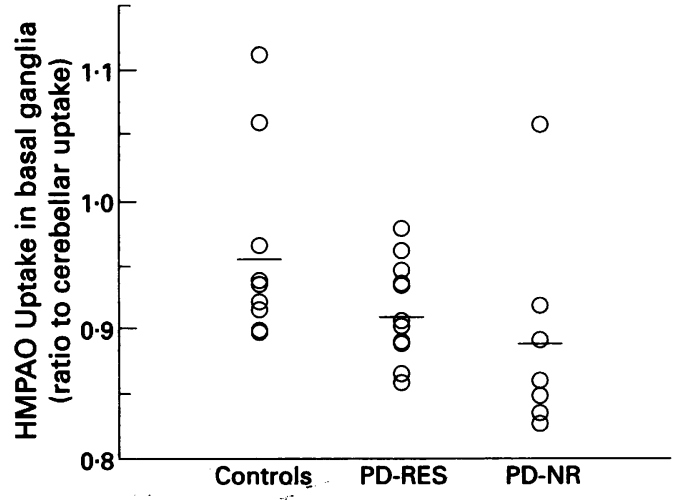

Figure HMPAO uptake in the basal ganglia (putamen and caudate) for 11 normal controls and 21 subjects with Parkinson's disease. The subjects with Parkinson's disease are divided into responders (PD-RES) and non-responders (PD-NR) to dopaminergic treatment. Values are the mean values from the two hemispheres. HMPAO uptake is expressed as the ratio to cerebellar uptake.

\section{SEMIQUANTITATIVE ANALYSIS \\ Subcortical regions}

Blood flow in the basal ganglia was decreased in the Parkinson's disease group by $5.23 \%$ compared with the control group $(p<$ $0.004)$, with the putamen $(5.58 \% ; \mathrm{p}<0.004)$ being slightly more affected than the caudate $(5.08 \% ; p<0.009)$. This reduction was seen in responders and non-responders. There was, however, an overlap between values for individual subjects in the three groups (figure). By contrast there was no difference in regional blood flow in the thalamus or brainstem in either the Parkinson's disease group as a whole, or in the responder or non-responder subgroups.

\section{Cortical regions}

A striking difference was noted in cortical blood flow between the responders and nonresponders with Parkinson's disease. Table 1 shows regional cerebral blood flow expressed as the ratio to mean cerebellar regional blood flow, for normal subjects, all Parkinson's disease groups, and the responder and nonresponder subgroups. In the Parkinson's

\begin{tabular}{lllll}
\hline $\begin{array}{l}\text { Anterior } \\
\text { parietal }\end{array}$ & $\begin{array}{l}\text { Posterior } \\
\text { parietal }\end{array}$ & Occipital & $\begin{array}{l}\text { Medial } \\
\text { temporal }\end{array}$ & $\begin{array}{l}\text { Lateral } \\
\text { temporal }\end{array}$ \\
\hline 0.780 & 0.799 & 0.999 & 0.777 & 0.786 \\
$(0.052)$ & $(0.039)$ & $(0.067)$ & $(0.040)$ & $(0.040)$ \\
0.747 & 0.734 & 0.976 & 0.746 & 0.756 \\
$(0.036)$ & $(0.039)$ & $(0.057)$ & $(0.051)$ & $(0.042)$ \\
0.753 & 0.745 & 0.986 & 0.765 & 0.767 \\
$(0.034)$ & $(0.037)$ & $(0.049)$ & $(0.037)$ & $(0.028)$ \\
0.734 & 0.713 & 0.957 & 0.708 & 0.735 \\
$(0.039)$ & $(0.034)$ & $(0.067)$ & $(0.055)$ & $(0.057)$ \\
& & & & \\
0.004 & 0.0001 & NS & 0.018 & 0.008 \\
NS & 0.0001 & NS & NS & NS \\
0.009 & 0.0001 & NS & 0.0005 & 0.003 \\
NS & 0.04 & NS & 0.002 & NS \\
\hline
\end{tabular}

disease responder group for most regions the ratio of regional cerebral blood flow to cerebellar regional blood flow was not significantly different from controls, with only three regions showing significant reductions: posterior parietal (by $6.76 \%$; $<0.001$ ), superior parietal $(6.31 \% ; \mathrm{p}<0.005)$, and posterior frontal $(3.61 \% ; p<0.02)$. By contrast, in the non-responders a significant decrease was seen in all cortical regions except occipital and inferior parietal cortex-namely, prefrontal cortex $(7.02 \% ; \mathrm{p}<0.004)$, anterior frontal cortex $(7 \cdot 11 \% ; \mathrm{p}<0.002)$, posterior frontal cortex $(6.72 \% ; p<0.004)$, superior parietal cortex $(8.38 \% ; \mathrm{p}<0.002)$, anterior parietal cortex $(5.90 \% ; \mathrm{p}<0.009)$, posterior parietal cortex $(10.76 \%$; $<0.0001)$, medial temporal cortex $(8.88 \% ; \mathrm{p}<0.0005)$, and lateral temporal cortex $(6.49 \%$; $<<0.003)$. Regional cerebral blood flow was significantly lower in the non-responders than in the responders in the medial temporal cortex $(7.45 \% ; p<0.002)$, posterior parietal cortex $(4.30 \% ; p<0.04)$, and pre-frontal cortex $(5.00 \% ; \mathrm{p}<0.05)$

To determine whether this reduction was symmetrical or asymmetrical absolute asymmetry ratios between the hemispheres were calculated for each cortical area, and the mean difference for each area, for each group, was compared between groups. There was no difference between ratios for any region between controls, responders, and nonresponders.

It has been suggested that the cortical changes represent functional changes consequent on the loss of subcortical-cortical connections. If this is the case asymmetrical cortical involvement might be expected in patients with clinically asymmetrical disease. To test this hypothesis in responders with asymmetrical Parkinson's disease (12 subjects), cortical regional blood flow in the hemisphere contralateral to the affected side was compared with that in the hemisphere ipsilateral to the deficit. No difference was found for any cortical region. Values in particular cortical areas showing reduced blood flow (side contralateral to clinically affected side first) included the prefrontal cortex $(0.742(0.045) \quad v \quad 0.747(0.052)$; anterior frontal cortex $0.745(0.038) v 0.745(0.047)$; posterior frontal cortex $(0.752(0.040) v$ $0.751 \quad(0.048)$; posterior parietal cortex $(0.745(0.032)$ v $0.757(0.039)$; superior parietal cortex $(0.779 \quad(0.055) \quad v \quad 0.764$ (0.051). All comparisons were NS.

\section{Changes after treatment with levodopa}

Mean regional blood flow in the putamen (ratio to cerebellar blood flow) in the patients with Parkinson's disease, increased by $3 \cdot 17 \%$ $(p<0.001)$ after treatment with levodopa. This increase was similar in both responders (3.03\%; $\mathrm{p}<0.007)$ and non-responders (3.46\%; $p<0.077)$; the lack of significance in the non-responders was due to the smaller number in this group. No significant changes were seen in either the responders or nonresponders in the caudate, other subcortical 
regions, or in any cortical regions after treatment with levodopa.

Asymmetry in basal ganglia regional blood flow has been reported in Parkinson's disease, and it has been suggested that it is reduced by levodopa. In our patients with Parkinson's disease mean (SD) basal ganglia asymmetry was reduced after levodopa (from 4.87 (3.50) to $2.40(1.52) ; \mathrm{p}<0.006)$; this reduction was seen in both responders and non-responders $(4.75(3.53)$ to $2.11(1.30)$ and $5.09(4.97)$ to $2.99(1.86)$ respectively). A decrease in basal ganglia asymmetry after treatment with levodopa was seen in 11 of the 14 responders and in six of the seven non-responders.

\section{Discussion}

Our results show that in Parkinson's disease, even early in the course of the disease, HMPAO SPECT indicates a significant decrease in regional blood flow in the basal ganglia. This is consistent with previous PET studies. ${ }^{4}$ An early HMPAO SPECT study failed to show any consistent alteration in blood flow in the basal ganglia in 16 parkinsonian patients. ${ }^{17}$ The patients were on treatment with levodopa, however, and basal ganglia uptake was assessed only visually and hence bilateral and symmetrical reductions in uptake may have been underestimated. A study with semiquantitative analysis showed increased asymmetries in basal ganglia uptake in patients with Parkinson's disease, particularly those with advanced disease. ${ }^{14}$ Mean blood flow in the basal ganglia was lower in patients with untreated Parkinson's disease than in controls but this difference did not reach significance. ${ }^{14}$ These two studies used single headed gamma cameras. ${ }^{1417}$ With the three headed high resolution camera used in our study we found significantly reduced regional blood flow in the basal ganglia, but there was an overlap between subjects with Parkinson's disease and controls (fig 1); therefore the specificity of this finding is not sufficient to provide useful diagnostic information.

Levodopa increases global cerebral blood flow, ${ }^{18}$ but less is known about its effect on regional cerebral blood flow. PET studies have shown that it may increase blood flow in the basal ganglia to a greater extent than flow in other regions, ${ }^{8}$ and this finding is supported by an HMPAO SPECT study in a small group of subjects. ${ }^{7}$ This approach might provide useful diagnostic information. We looked at basal ganglia regional blood flow by comparing HMPAO uptake in the basal ganglia with that in the cerebellum. Our results showed a significant relative increase in blood flow in the basal ganglia, and more specifically in the putamen, after treatment with levodopa. The relative increase was similar in both responders and non-responders; therefore this change is likely to be non-specific and certainly does not correlate with dopaminergic responsiveness. We did not compare changes before and after levodopa with those in normal subjects in order to reduce radiation dose in these subjects, but PET studies suggest that normal controls also show a relative rise in basal ganglia blood flow after levodopa although it may be to a lesser extent than that seen in patients with Parkinson's disease.$^{8}$ Our results suggest that this approach will not prove useful diagnostically.

A striking finding in our patients with Parkinson's disease was of areas of pronounced reduction in cortical blood flow and this was strikingly more common in nonresponders than in responders. This global fall could result from the functional effects of loss of diffuse cortical connections, such as those from the nucleus basalis of Meynert, ${ }^{19}$ or from cortical pathology. The first explanation was suggested after the finding of reduced regional blood flow in the frontal cortex contralateral to the clinical deficit. ${ }^{4} \mathrm{We}$ were unable to show any difference, however, in any cortical region contralateral to the worst side in our patients with asymmetrical disease. This favours a direct pathological involvement of the cerebral cortex. Areas particularly affected were the prefrontal cortex, frontoparietal regions, and medial temporal cortex. A highly significant reduction in medial temporal blood flow was seen in nonresponders compared with responders. This pattern is similar to that seen in Alzheimer's disease, ${ }^{720}$ and in Parkinson's disease complicated by dementia, ${ }^{20}$ although in our study no subjects had clinical dementia. This suggests additional cortical pathology in these patients. All our patients with Parkinson's disease fulfilled clinical criteria for idiopathic Parkinson's disease; however, as previously mentioned, as many as $25 \%$ of such subjects may not have typical neuropathological findings of Parkinson's disease on postmortem examination. ${ }^{10} \mathrm{~A}$ pronounced improvement in clinical state, particularly bradykinesia and rigidity, after levodopa increases the likelihood that the neuropathological diagnosis is indeed idiopathic Parkinson's disease. ${ }^{9}$ Therefore, it is likely that the responders represent idiopathic Parkinson's disease pathologically. Possible causes of the cortical changes seen in non-responders, which may be different for individual subjects, include Alzheimer's pathology presenting in a preclinical dementia state, progressive supranuclear palsy, and diffuse Lewy body disease. Functional imaging in progressive supranuclear palsy shows considerable cortical involvement, particularly in the frontal lobes, but also in other cortical regions including the temporal cortex. ${ }^{21}$ Most patients with Parkinson's disease have cortical Lewy bodies, ${ }^{10}$ but a subgroup seem to have more numerous involvement and may show less dopaminergic responsiveness. ${ }^{22}$ Cortical Lewy bodies are numerous in the temporal cortex, an area with particularly reduced HMPAO uptake in the non-responders in our study. The appearances of cortical regional blood flow in subjects with diffuse Lewy body disease is not yet known. The symmetrical cortical involvement seen in the non-responders, 
as shown by no increase in the asymmetry ratios between hemispheres, suggests that vascular processes leading to multi-infarct dementia and a parkinsonian syndrome does not account for the changes seen, Similarly the cortical changes do not represent those seen in other possible differential diagnoses such as corticobasal degeneration ${ }^{23}{ }^{24}$ or multiple system atrophy; in the last the cortex is spared. What remains striking, however, is that even in the earliest clinical stages (most of the subjects were studied at presentation) pronounced cortical abnormalities are present in the dopaminergic non-responders suggesting additional cortical involvement. Therefore, HMPAO SPECT scanning early in the course of the disease may give useful diagnostic and pathophysiological information in parkinsonian syndromes. It should be remembered that although the distribution of HMPAO is a good approximation to regional cerebral blood flow, ${ }^{5}$ and less isotope will be distributed to regions of low perfusion, retention in such areas will be greater, and therefore the patterns revealed on HMPAO SPECT represent maps of isotope uptake. Furthermore, HMPAO uptake is an active process and the uptake deficits we detected may reflect impaired intracellular retention of HMPAO as well as reduced regional cerebral blood flow. ${ }^{25}$ Nevertheless the cortical abnormalities we describe may allow identification at an early stage of patients who will respond poorly to treatment and possibly later develop an atypical disease. Prolonged clinical follow up and eventual neuropathological correlation is required to determine the clinical course and pathological cause of these cortical deficits.

1 Shoulson I and the Parkinson Study group. Effect of deprenyl on the progression of disability in early Parkinson's disease. N Engl ₹ Med 1989;321:1364-71.

2 Martin WRW, Stoessl AJ, Adam AJ, et al. Positron emission tomography in Parkinson's disease: Glucose and dopa metabolism. Adv Neurol 1986;45:95-8.

3 Brooks DJ, Ibanez V, Sawle GV, et al. Differing pattern of striatal ${ }^{18} \mathrm{~F}$-dopa uptake in Parkinson's disease, multiple system atrophy, and progressive supranuclear palsy. Ann Neurol 1990;28:547-55.

4 Wolfson LI, Leenders KL, Brown LI, Jones T. Alterations of regional cerebral blood flow and oxygen metabolism in Parkinson's disease. Neurology 1985;35:1399-405.
5 Neirinckx RD, Canning LR, Piper IM, et al. Technetium99m d,1-HM-PAO: a new radiopharmaceutical for SPECT imaging of regional cerebral blood perfusion. f Nucl Med 1987;28:191-202.

6 Kouris K, Jarritt PH, Costa DC, Ell PJ. Physical assessment of the GE/GCR Neurocam and comparison with a single rotating gamma-camera. Eur $\mathcal{f}$ Nucl Med 1992; 19:236-42.

7 Costa DC, Ell PJ, Philpot M, Levy R. CBF tomograms with [ $\left.{ }^{99 m} \mathrm{Tc}\right]-\mathrm{HM}-\mathrm{PAO}$ in patients with dementia (Alzheimer type and HIV) and Parkinson's diseaseinitial results. $f$ Cereb Blood Flow Metab 1988;8: initial resul15.

8 Leenders KL, Wolfson L, Gibbs JM, et al. The effects of L-dopa on regional cerebral blood flow and oxygen metabolism in patients with Parkinson's disease. Brain 1985;108:171-91.

9 Koller WC. How accurately can Parkinson's disease be diagnosed? Neurology 1992;42(suppl 1):6-16.

10 Hughes AJ, Daniel SE, Kilford L, Lees AJ. Accuracy of clinical diagnosis of idiopathic Parkinson's disease: a clinico-pathological study of 100 cases. $\mathcal{f}$ Neurol Neurosurg Psychiatry 1992;55:181-4.

11 Kempster PA, Frankel JP, Bovington M, Webster R, Lees AJ, Stern GM. Levodopa peripheral pharmokinetics and duration of motor response in Parkinson's disease. $\mathcal{f}$ Neurol Neurosurg Psychiatry 1989;52:718-23.

12 Hughes AJ, Lees AJ, Stern GM. Apomorphine test to predict dopaminergic responsiveness in parkinsonian predict dopaminergic responsive

13 Talairach J, Tournoux P. Co-planar stereotaxic atlas of the human brain. New York: Thieme Medical Publishers, 1988.

14 Pizzolato G, Dam M, Borsato N, et al. ${ }^{99 m}$ Tc-HM-PAO SPECT in Parkinson's disease. $f$ Cereb Blood Flow Metab 1988;8:S101-8.

15 Spampinato U, Habet MO, Mas JL, et al. (99Tc)-HMPAO SPECT and cognitive impairment in Parkinson's disease: a comparison with dementia of the Alzheimer type. F Neurol Neurosurg Psychiatry 1991;54:787-92.

16 Hoehn MM, Yahr MD. Parkinsonism: onset, progression and mortality. Neurology 1967;17:427-42.

17 Smith FW, Gemmell HG, Sharp PF, Besson JAO. Technetium-99m HMPAO imaging in patients with basal ganglia disease. $B r \mathcal{F}$ Radiol 1988;61:914-20.

18 Granerus A, Nilsson NJ, Suurkula $M$, Svanborg A Cerebral blood flow in Parkinson's syndrome. In: Hassler RG, Christ J. eds. Advances in Neurology. Vol 40. New York: Raven Press, 1984:403-5.

19 Brooks DJ, Frackowiak RSJ. PET and movement disorders. F Neurol Neurosurg Psychiatry. 1989;(special suppl): 68-77.

20 Frackowiak RSJ, Pozzilli C, Legg NJ, et al. Regional cerebral oxygen supply and utilisation in dementia. A clini$\mathrm{cal}$ and physiological study with oxygen-15 and positron tomography. Brain 1981;104:753-8.

21 Blin J, Baron JC, Dubois B, et al. Positron emission tomography study in progressive supranuclear palsy. Arch Neurol 1990;47:747-52.

22 Gibb WRG. Neuropathology in movement disorders. f Neurol Neurosurg Psychiatry 1989;(special suppl): 55-67.

23 Sawle GV, Brooks DJ, Marsden CD, Frackowiak RSJ. Corticobasal degeneration. A unique pattern of regional hypometabolism and striatal fluorodopa uptake demonstrated by positron emission tomography. Brain 1991;114:541-56.

24 Markus HS, Costa D, Lennox G, Marsden C, Lees A. Tc-99m-HMPAO regional cerebral blood flow single photon emission tomography in corticobasal degeneration. $\mathcal{f}$ Neurol 1992;239:S107.

25 Neirinckx RD, Burke JF, Harrison RC, Forster AM, Andersen A R, Lassen N A. The retention mechanism of technetium-99m-HM-PAO: intracellular reaction with glutathione. f Cereb Blood Flow Metab 1988;8:S4- 